\title{
A decentralized spectrum allocation and partitioning scheme for a two-tier macro- femtocell network with downlink beamforming
}

\author{
Sunheui Ryoo ${ }^{1}$, Changhee Joo ${ }^{2^{*}}$ and Saewoong Bahk
}

\begin{abstract}
This article examines spectrum allocation and partitioning schemes to mitigate cross-tier interference under downlink beamforming environments. The enhanced SIR owing to beamforming allows more femtocells to share their spectrum with the macrocell and accordingly improves overall spectrum efficiency. We first design a simplified centralized scheme as the optimum and then propose a practical decentralized algorithm that determines which femtocells to use the full or partitioned spectrum with acceptable control overhead. To exploit limited information of the received signal strength efficiently, we consider two types of probabilistic femtocell base station (HeNB) selection policies. They are equal selection and interference weighted selection policies, and we drive their outage probabilities for a macrocell user. Through performance evaluation, we demonstrate that the outage probability and the cell capacity in our decentralized scheme are significantly better than those in a conventional cochannel deployment scheme. Furthermore, we show that the cell utility in our proposed scheme is close to that in the centralized scheme and better than that in the spectrum partitioning scheme with a fixed ratio.
\end{abstract}

\section{Introduction}

The deployment of femtocells is generating attention among mobile operators as a cost-effective and highbandwidth solution for next generation wireless networks. A femtocell is a low power, short range data access point that enhances indoor coverage, while backhauling incoming traffic over the IP network. Operating in the licensed spectrum owned by a mobile operator, femtocells provide mobile convergence services through the broadband backhaul in long-term evolution (LTE) networks. Their benefits include capacity gain, good coverage, and reduced battery consumption of handsets [1].

The coexistence of macrocell and femtocell networks at a same spectrum, however, incurs additional control challenges due to the cross-tier interference between macrocell and femtocells as well as the co-tier interference. Although the co-tier interference control has been extensively studied in the literature [2-7], the cross-tier interference control still has been an open problem and remains as a key technical challenge $[1,8]$.

\footnotetext{
* Correspondence: cjoo@unist.ac.kr

${ }^{2}$ School of Electrical and Computer Engineering, UNIST, Ulsan, Korea Full list of author information is available at the end of the article
}

Previous studies on a two-tier network investigated the uplink capacity in overlaid macro-cell/microcell code division multiple access (CDMA) systems, assuming that the operator plans microcell deployment $[9,10]$. This assumption may not be acceptable for selfdeployed femtocell networks [8].

One of the key technical implementation issues in a two-tier network is managing the cross-tier interference between existing macrocell and femtocell networks $[1,8]$. To ensure minimal impact on the performance of the existing macrocell network, femtocells should be designed so as to limit the low level interference $[11,12]$. With respect to the cross-tier interference, the throughput performance of cochannel deployment of femtocells and macrocells has been thoroughly investigated through simulations in [13]. Assigning diffierent spectrums to macrocell and femtocell networks, respectively, can be a solution to the cross-tier interference mitigation [14]. However, considering the scarcity of radio resources and the difficulty in spectrum allocation, it is preferable to use the same spectrum.

With the goal of improving performance of both macrocell and femtocell users under cochannel deployment, the MIMO beam subset selection strategy at the 
microcell has been proposed in [15]. It maximizes the throughput of the macrocell by optimizing the trade-off between multiplexing gain and multiuser interference, and it also suppresses the cross-tier interference with a reduced number of beams. In [16], authors solve the beamforming optimization problems which are total transmit power minimization problem, mean-square error balancing problem and interference power minimization problem. A centralized beamforming strategy that adaptively changes beam patterns and controls the total transmit power of cells is proposed in [17]. However, these cochannel deployments are still exposed to the cross-tier interference problem.

The hybrid spectrum usage deals with system driven criteria to decide inner and outer regions analytically $[18,19]$. Femtocells embedded in the macrocell are classified into inner and outer femtocells according to the distance threshold, which operate in partitioned spectrum and shared spectrum, respectively. The interference limited coverage area of a cochannel HeNB is calculated in [20]. Femtocells only in the outer region are allowed to operate in the cochannel operation for more efficient cell search. On the other hand, femtocells in the inner region use a partitioned spectrum. However, they do not use the optimal division ratio between shared and partitioned spectrum, though they require centralized coordinators which induce some cross-tier control overhead and delay.

Our article mainly investigates a decentralized algorithm and probabilistic HeNB selection policies under hybrid spectrum usage with beamforming transmission. The objective of this article is to develop spectrum allocation and partitioning schemes to mitigate cross-tier interference. Our focus is on the following questions:

- What is the effect of the beamforming gain on the user's SIR, interference mitigation and spectrum efficiency? What is the optimal division ratio between shared and partitioned spectrum to maximize the total cell utility?

- What is a simplified centralized solution for the spectrum allocation and partitioning problem? What is a practical decentralized one that requires low complexity and minimal cross-tier feedback?

- What is the outage probability for a macrocell user when a probabilistic HeNB selection policy is applied with the limited feedback information of received signal strength?

Before we answer these questions, we first provide a system model for a two-tier network and a simplified mathematical model for beamforming transmission in Section 2. Using this model, we then derive an optimal spectrum partitioning ratio to maximize the cell utility, and propose a simplified centralized algorithm in Section 3. To make our design practical, we propose a decentralized algorithm and analyze its outage probability for a macro user in Section 4, and evaluate the performance in Section 5. Finally, we conclude our article in Section 6.

\section{System model}

We consider a two-tier network that consists of a macrocell network and multiple femtocell networks. The macrocell network has a macrocell user (MUE) and a single base station (MeNB) that covers a cell radius of $R_{m}$. The macrocell network is overlaid with femtocell networks, each with radius $R_{f}\left(R_{f}<R_{m}\right)$, where $\mathbf{K}_{\mathbf{t}}$ denotes a set of femtocell base stations (HeNBs). We consider femtocells provide closed access to a fixed set of subscribed indoor users within radio range who are licensed to use the femtocell for privacy and security reasons. Each HeNB $i$ is assumed to have an associated femtocell user (HUE $i$ ).

Femtocells are deployed over the existing macrocell network and share the same frequency spectrum with macrocells. Due to spectral scarcity, the femtocells and macrocells have to reuse the allocated frequency band partially or totally which leads to co-tier or cross-tier interference. The co-tier interference is caused by neighboring femtocells and the cross-tier interference occurs between femtocell and macrocell networks. For simplicity, we will ignore background thermal noise since the noise contribution is minimal in an interference-limited network. We focus on mitigating the cross-tier interference between the macrocell and femtocells in downlink by partitioning severe interfering femtocells.

We utilizes the beamforming transmission which enhances the strength of the desired signal and reduces the interference. We assume that base stations (MeNB and HeNBs) are equipped with beamforming antennas, while user equipments (MUE and HUEs) are not due to their physical device limitations.

\subsection{Beamforming gain on the SIR in a two-tier network}

We investigate the effiect of beamforming on the SIR gain in a two-tier femtocell network. To do so, we use a simplified beamforming antenna model in [21], where the signal propagation area is divided into two areas according to the beam gain: main lobe and side lobe areas as shown in Figure 1. We denote the received signal strength at a receiver by $S^{R}(\theta)$, which can be expressed as

$$
S^{R}(\theta)=\left\{\begin{array}{l}
g_{m} P^{R}, \text { if } \theta \leq \theta_{m} \\
g_{s} P^{R}, \text { otherwise, }
\end{array}\right.
$$




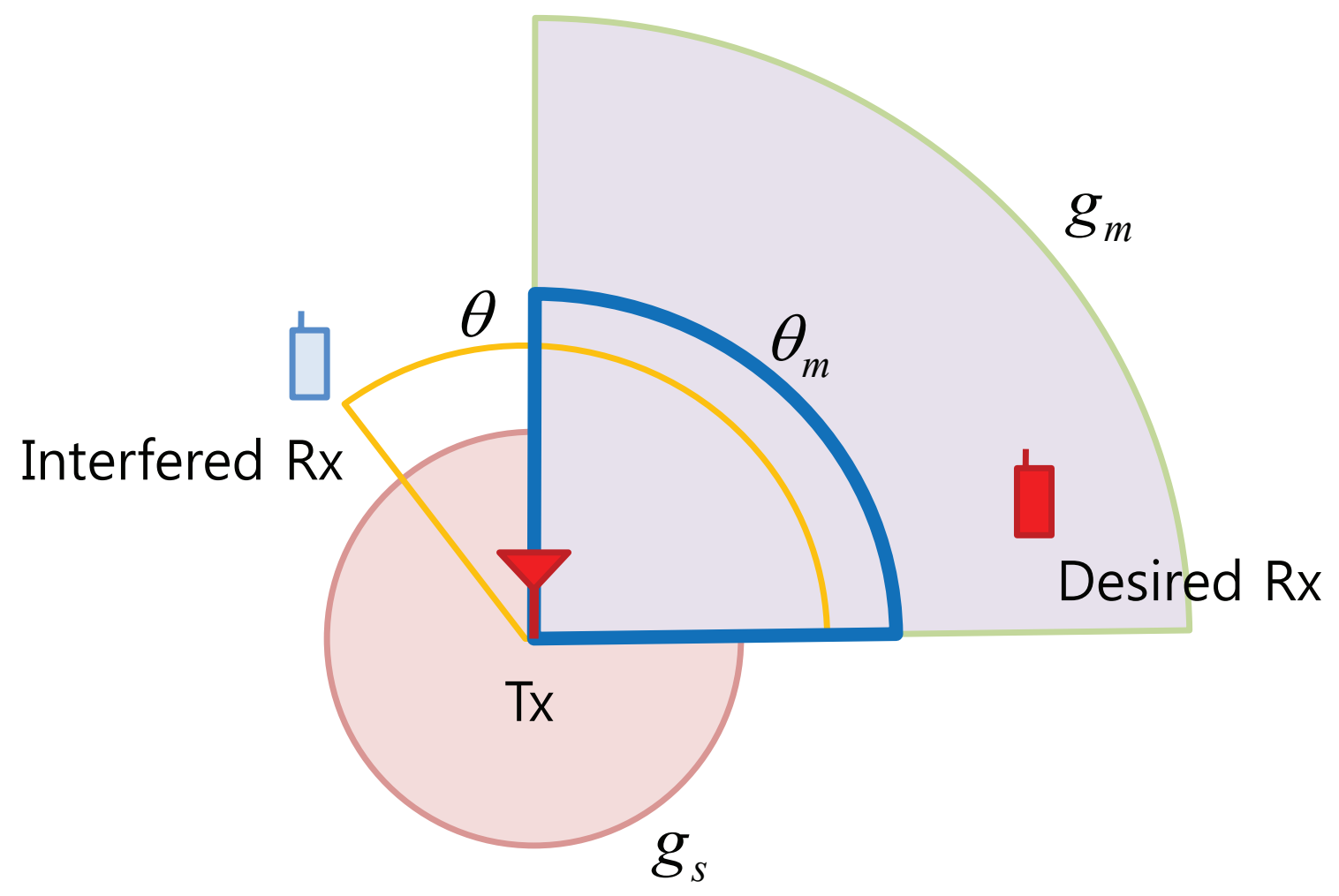

Figure 1 Simplified model for the beamforming gain with main and side lobes.

where $\theta$ denotes the antenna alignment angle between a transmitter and a receiver, and $P^{R}$ denotes the received signal strength from omnidirectional transmission. Let $\theta_{m}$ denotes the beamwidth of the main lobe. The main lobe covers the range of angle $\theta_{m}$ with gain $g_{m}$, and side lobe covers the remaining range of angle $\left(2 \pi-\theta_{m}\right)$ with gain $g_{s}$. With beamforming transmission, the received signal strength depends on not only the channel attenuation and fading, but also the beamforming antenna alignment.

We assume perfect antenna control, where the beamforming transmission is directed toward the target receiver and achieves the antenna gain $g_{m}$. For side lobe beams, the interference is suppressed by the side beam gain $g_{s}$, i.e., $g_{s} \ll 1 \ll g_{m}$. When the transmitter uses beamforming, let $\gamma_{m}$ and $\gamma_{f_{i}}$ denote the received SIRs at MUE and HUE $i$, respectively. We assume optimal power control, where the received power at MUE and HUE is kept at $P^{R}$.

We calculate the average SIR gain at HUE of the beamforming transmission over the omnidirectional transmission. We obtain the average SIR at HUE $i$ with beamforming transmission, $\mathrm{E}\left[\gamma_{f_{i}}\right]$, which is the ratio of the desired signal strength to the cross-tier interference as

$$
\mathrm{E}\left[\gamma_{f_{i}}\right]=\mathrm{E}\left[\frac{g_{m} P^{R}}{\gamma_{i} I_{i}^{m}}\right]
$$

where $I_{i}^{m}$ denote the cross-tier interference from MeNB to HUE $i$ under omnidirectional transmission, and $y_{i}$ denotes the beamforming gain indicator with $y_{i}=$ $g_{m}$, if HUE $i$ is located in the main lobe area of MeNB, and $y_{i}=g_{s}$, otherwise. Assuming that UEs and HeNBs are randomly distributed, the probability that $\mathrm{HUE}_{i}$ is located in the main lobe of MeNB is $\frac{1}{N_{b}}$, and thus we can calculate average antenna gain $E\left[y_{i}\right]$ as $\mathrm{E}\left[y_{i}\right]=\frac{1}{N_{b}} g_{m}+\frac{N_{b}-1}{N_{b}} g_{s}$. Then it can be easily shown that the average SIR gain at a HUE of with and without beamforming transmissions as

$$
\Psi\left(N_{b}\right) \geq \frac{g_{m} N_{b}}{\left(N_{b}-1\right) g_{s}+g_{m}} .
$$

where $\Psi\left(N_{b}\right)$ denotes the average gain of beamforming transmission under configuration $N_{b}$ over the omnidirectional transmission, and satisfies $\Psi\left(N_{b}\right)=\mathrm{E}\left[\frac{g_{m}}{\gamma_{i}}\right] \geq \frac{g_{m}}{\mathrm{E}\left[y_{i}\right]}$ by Jensen's inequality.

Similarly, we calculate the average SIR gain at MUE with and without the beamforming transmission. The 
average SIR at MUE with beamforming transmission, which is the ratio of the desired signal strength to the sum of cross-tier interference from HeNBs can be written as

$$
\mathrm{E}\left[\gamma_{m}\right]=\mathrm{E}\left[\frac{g_{m} P^{R}}{\sum_{i \in \mathbf{K}_{\mathrm{s}}} x_{i} I_{i}^{f}}\right],
$$

where $\mathbf{K}_{\mathbf{s}}$ is the set of HeNBs which use the full spectrum, and generate cross-tier interference to MUE, and $I_{i}^{f}$ denotes the cross-tier interference at MUE due to the omnidirectional transmission from HeNB $i$. Let $x_{i}$ denotes the beamforming gain indicator which $x_{i}$ can be determined based on the location of MUE with respect to the beamforming transmission of HeNB $i$. Thus, we notice the average SIR gain $\Psi\left(N_{b}\right)$ at MUE of the beamforming transmission over the omnidirectional transmission is identical to that at HUE as shown in (3).

This average SIR gain $\Psi\left(N_{b}\right)$ shows how much average SIR at UEs is enhanced by beam-forming transmission in the two-tier network. This enhancement of SIR comes from the desired signal reinforcement and cross-tier interference mitigation. The lager $N_{b}$ means the lager $g_{m}$ and smaller $g_{s}$, and thus the average SIR gain $\Psi\left(N_{b}\right)$ owing to beamforming gain increases with the larger $N_{b}$. We summarize the SIR gains in the two-tier network according to beamforming transmission in Table 1.

\section{Optimum ratio of spectrum partitioning and centralized algorithm}

The cross-tier interference caused by nearby active users can lead to unacceptable performance degradation in heterogeneous femto-macrocell networks. To minimize the interference, the spectrum is divided into the shared and partitioned spectrums. By the partitioned spectrum usage, the cross-tier interference becomes lower, but the amount of available spectrum becomes also smaller. By the shared spectrum usage, each user can have a more amount of spectrum but suffers from higher cross-tier interference. The hybrid spectrum usage to exploit the merits of the two types of spectrums can be considered too. When UEs locates near active cross-tier transmitter, i.e., an MUE located near active HeNBs, or HUEs located near MeNB, they may experience significant cross-tier interference. To avoid this, the spectrum needs to be divided into two parts. A fraction of it,

Table 1 Number of beams and beamforming gain

\begin{tabular}{ccccc}
\hline $\boldsymbol{N}_{\boldsymbol{b}}$ & $\boldsymbol{\theta}_{\boldsymbol{m}}$ & $\boldsymbol{g}_{\boldsymbol{m}}$ & $\boldsymbol{g}_{\boldsymbol{s}}$ & $\boldsymbol{\Psi}_{\boldsymbol{m}}, \boldsymbol{\Psi}_{\boldsymbol{f}}(\mathrm{dB})$ \\
\hline 1 & $2 \pi$ & 0.00 & 0.00 & 0.00 \\
4 & $\pi / 2$ & 9.84 & -30.00 & 6.02 \\
8 & $\pi / 4$ & 18.37 & -30.00 & 9.03 \\
\hline
\end{tabular}

denoted by shared spectrum, is used by both the macrocell and non-interfering femtocell networks. The rest, denoted by partitioned spectrum, is exclusively allocated to the femtocell networks.

In this article, we consider two problems to mitigate the cross-tier interference. One is the spectrum allocation problem that deals with determining which HeNBs should use the partitioned spectrum. The other is the spectrum partitioning problem that handles how much spectrum should be allocated for the shared and partitioned spectrums, respectively. To achieve optimal spectrum allocation, we need the channel feedback from each HeNB $i$, which we will discuss in detail in the Section 3.2. Before doing this, we analytically drive the optimal spectrum partitioning ratio $v_{p}^{*}$ as a function of the number of femtocells which use partitioned spectrum, | $\mathbf{K}_{\mathbf{p}} \mid$.

\subsection{Optimal ratio of spectrum partitioning}

We formulate the spectrum partitioning problem as a utility maximization problem that considers both spectrum efficiency and fairness. Let $\mathbf{K}_{\mathbf{p}}$ denotes the set of HeNBs that use the partitioned spectrum from the macrocell. Then we express the total cell utility, denoted by $U$, as the sum of each user's utility, which is a logarithmic function of the achieved link rate [22]:

$$
U=w_{m} \log C_{m}+w_{f} \sum_{i \in \mathbf{K}_{\mathbf{s}}} \log C_{f_{i}}+w_{f} \sum_{j \in \mathbf{K}_{\mathbf{p}}} \log C_{f_{j}}
$$

where $w_{m}$ and $w_{f}$ are relative weights for macrocell and femtocell links, respectively. $C_{m}, C_{f_{i}}$, and $C_{f_{j}}$ represent the link rates of MeNB, HeNB $i \in \mathbf{K}_{\mathbf{s}}$, and $\mathrm{HeNB} j$ $\in \mathbf{K}_{\mathbf{p}}$, respectively. Using the Shannon capacity formula for each link rate, i.e., $C_{m}$ : $=v_{s} \log \left(1+\gamma_{m}\right)$, $C_{f_{i}}:=\log \left(1+\gamma_{f_{i}}\right)$, and $C_{f_{j}}:=v_{p} \log \left(1+\gamma_{f_{j}}\right)$, where $v_{s}$ and $v_{p}\left(=1-v_{s}\right)$ are the ratios of the shared and partitioned spectrums, respectively, we can rewrite (5) as

$$
\begin{aligned}
U & =w_{m} \log v_{s}+w_{f}\left|\mathbf{K}_{\mathbf{p}}\right| \log \left(1-v_{s}\right) \\
& +w_{m} \log \left(\log \left(1+\gamma_{m}\right)\right)+w_{f} \log \left(\prod_{i \in \mathbf{K}_{\mathrm{s}}} \log \left(1+\gamma_{f_{i}}\right)\right) \\
& +w_{f} \log \left(\prod_{j \in \mathbf{K}_{\mathbf{p}}} \log \left(1+\gamma_{f_{j}}\right)\right)
\end{aligned}
$$

Let $v_{s}^{*}$ denotes an optimum ratio between the shared and partitioned spectrums that maximizes the utility $U$, i.e.,

$$
v_{s} *=\underset{v_{s}}{\arg \max } U .
$$


Since the utility is a concave function, the optimal $v_{s}^{*}$ should satisfy $\frac{d U}{d v_{s}}=0$. From

$$
\begin{aligned}
& \frac{d}{d v_{s}}\left\{w_{m} \log v_{s}+w_{f}\left|\mathbf{K}_{\mathbf{p}}\right| \log \left(1-v_{s}\right)+Q\right\} \\
& \quad=\frac{w_{m}}{v_{s}}-\frac{w_{f}\left|\mathbf{K}_{\mathbf{p}}\right|}{\left(1-v_{s}\right)}
\end{aligned}
$$

where $Q$ denotes the terms that are not associated with $v_{s}$, we can obtain (7), which is reversely proportional to the number of femtocells in the partitioned spectrum, $\left|K_{\mathbf{p}}\right|$.

$$
v_{s}^{*}=\frac{w_{m}}{w_{f}\left|\mathbf{K}_{\mathbf{p}}\right|+w_{m}} .
$$

The $v_{s}^{*}$ is reversely proportional to $\left|\mathbf{K}_{\mathbf{p}}\right|$. As it involves SIR and beamforming gain, $\left|\mathbf{K}_{\mathbf{p}}\right|$ is dependent on these factors $t$. We conduct numerical experiments under the setting that users are randomly located and equipped with three different beamforming gains, where the number of beams $N_{b}=2 \pi / \theta_{m}=1,4,8$. Table 2 provides the $v_{s}^{*}$ 's according to $N_{b}$ and the required SIR at MUE $\gamma_{m}^{q}$. With the stricter $\gamma_{m}^{q}$, the number of $\mathbf{K}_{\mathbf{p}}$ increases and thus the $v_{s}^{*}$ decreases. Because the cross-tier interference at UEs is suppressed by beamforming transmission, with the higher beamforming gain, i.e., $N_{b}=8$, the gap of $v_{s}^{*}$ with the different $\gamma_{m}^{q}$ becomes narrower than that of omnidirectional transmission, i.e., $N_{b}=1$.

\subsection{Centralized spectrum allocation and partitioning algorithm}

Ideally to mitigate cross-tier interference, there would be a central entity in charge of intelligently telling each cell which subchannels to use. This entity would need to collect information from the femtocells and their users, and use it to find an optimal or a good solution within a short period of time.

However, since the number and position of the femtocells are initially unknown due to the individualistic nature of the HeNBs, this approach poses some hard problems. The presence of hundreds of femtocells makes the optimization problem too complex, and latency issues arise when trying to facilitate the

Table 2 Optimal ratio $v_{s}^{*}$ for the shared spectrum with the number of beams and required SIR

\begin{tabular}{ccccccc}
\hline & \multicolumn{7}{c}{ Required SIR $\boldsymbol{\gamma}^{\mathrm{A}}(\mathbf{d B})$} \\
\cline { 2 - 7 } $\boldsymbol{N}_{\boldsymbol{b}}$ & $\mathbf{0}$ & $\mathbf{2}$ & $\mathbf{4}$ & $\mathbf{6}$ & $\mathbf{8}$ & $\mathbf{1 0}$ \\
\hline 1 & 0.69 & 0.61 & 0.51 & 0.37 & 0.22 & 0.02 \\
4 & 0.84 & 0.78 & 0.75 & 0.67 & 0.59 & 0.47 \\
8 & 0.86 & 0.84 & 0.80 & 0.75 & 0.69 & 0.59 \\
\hline
\end{tabular}

femtocell networks with the central subchannels broker throughout the backhaul.

Therefore we develop a simplified centralized solution for spectrum allocation and partitioning, which use minimal channel information between cross-tier networks. It aims to maximize the number of HeNBs that use the full spectrum $\left|\mathbf{K}_{\mathbf{s}}\right|$ while satisfying the SIR requirements of MUE and HUEs.

We assume that HeNBs communicate with MeNB through the wired backhaul network, which incurs control overhead and delay in message delivery. Between cross-tier networks, they can overhear each other's pilot signal. The spectrum allocation algorithm needs SIR measurement tests at HUE and HeNB, respectively. In the first test at each $\operatorname{HUE} i$, if its $\operatorname{SIR} \gamma_{f_{i}}$ is greater than $\gamma_{f}^{q}$, then the associated HeNB $i$ belongs to $\mathbf{F}_{\mathbf{1}}$, otherwise $\mathbf{F}_{2}$. This means that femtocell $i$ in $\mathbf{F}_{\mathbf{1}}$ is a candidate for spectrum sharing. Then HeNB $i$ performs the second test by measuring the signal strength received from the MUE's pilot signal. From this, it reversely estimates the interference from HeNB $i$ to MUE, i.e., $I_{i}$, and sends the result to MeNB which will determine which HeNBs belong to $\mathbf{K}_{\mathbf{s}}$.

Algorithm 1 describes the centralized spectrum allocation and partitioning algorithm, and Figure 2 presents the control signalling procedures, where solid lines represent wireless transmissions with beamforming or omnidirectional transmission, and dashed lines symbolize wired transmissions through the backhaul network.

$i$ MeNB transmits a pilot signal with beamforming. Each HUE $i$ performs the first test. Then, each HUE $i$ informs HeNB $i$ whether HeNB $i$ belongs to $\mathbf{F}_{\mathbf{1}}$ or not.

$i i$ For the second test, MUE transmits a pilot signal omnidirectionally with transmission power $P_{t}$. Only HeNB $i$ in $\mathbf{F}_{\mathbf{1}}$ measures the received signal strength, and reversely estimates its cross-tier interference at MUE, $i$. e., $I_{i}$, and sends the result to MeNB through the backhaul network.

iii MeNB sorts the HeNBs in the increasing order of $I_{i}$. That is, HeNB $j$ incurs less interference to MUE than HeNB $j+1$ for $j=1,2, \ldots,\left|\mathbf{F}_{1}\right|-1$. MeNB sums the interference from $\mathrm{HeNB} 1$ through $\operatorname{HeNB} j$, and we denote it as $S_{j}$. If $S_{j}$ is smaller than the permitted interference level, i.e., $P^{R} / \gamma_{m}^{q}$, MeNB decides to put HeNB $j$ into the set $\mathbf{K}_{\mathbf{s}}$, and increase $j$ by one. Otherwise, MeNB completes the selection process for $\mathbf{K}_{\mathbf{s}}$ and accordingly decides $\mathbf{K}_{\mathbf{p}}$. Then MeNB determines $v_{p}$ using $\left|\mathbf{K}_{\mathbf{p}}\right|$ and Equation (7).

$i v$ MeNB sends the $v_{p}$ to the HeNBs in $\mathbf{K}_{\mathbf{p}}$ via the backhaul network. HeNB $i$ in $\mathbf{K}_{\mathbf{p}}$ uses the partitioned spectrum, while HeNB $j$ in $\mathbf{K}_{\mathbf{s}}$ uses the full spectrum. The detailed algorithm is shown in Algorithm 1. 


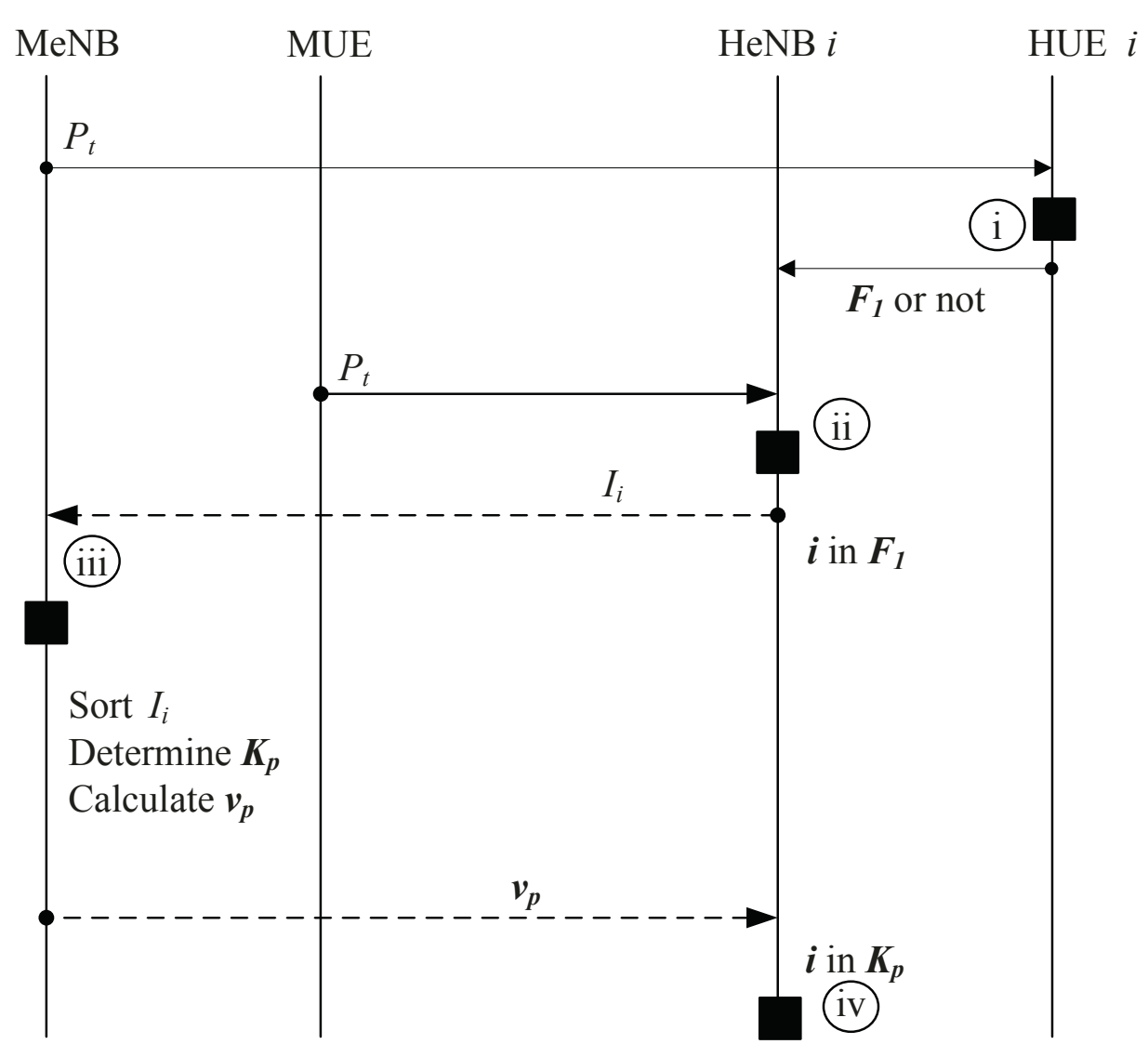

Figure 2 Control signalling procedures for centralized spectrum allocation and partitioning for a case of HeNB $i$ in $\mathrm{K}_{\mathrm{p}}$.

Algorithm 1. Centralized spectrum allocation and partitioning scheme

1: $i$ MeNB transmits a pilot with beamforming transmission.

2: for each HUE $i$ do

3: $\quad$ if $\gamma_{f_{i}}>\gamma_{f}^{q}$ then

4: $\quad$ HeNB $i \rightarrow \mathbf{F}_{\mathbf{1}}$

5: else

6: $\quad$ HeNB $i \rightarrow \mathbf{F}_{2}$

7: $\quad$ end if

8: end for

9: HeNB $i$ knows whether it belongs to $\mathbf{F}_{\mathbf{1}}$ or not from the HUE $i$ 's co-tier feedback.

10: $i$ MUE transmits a pilot signal omnidirectionally, and HeNB $i$ measures the received signal strength.

11: HeNB $i$ in $\mathbf{F}_{\mathbf{1}}$ sends reversely estimated $I_{i}$ to the MeNB via the backhaul network.

12: iii MeNB sorts HeNBs in the increasing order of $I_{i}$.

13: Let $j=1, S_{1}=I_{1}$

14: while $S_{j}<P^{R} / \gamma_{m}^{q}$ do

15: $\quad$ HeNB $j \rightarrow \mathbf{K}_{\mathbf{s}}$

16: $S_{j}=S_{j}+I_{j}$
17: $\quad j=j+1$

18: end while

19: $\mathbf{K}_{\mathbf{p}}=\mathbf{K}_{\mathbf{t}}-\mathbf{K}_{\mathbf{s}}$

20: MeNB determines $v_{p}$ using Equation (7).

21: iv MeNB informs the HeNBs in $\mathbf{K}_{\mathbf{p}}$ of the $v_{p}$ through the backhaul network.

22: if $\mathrm{HeNB} i \in \mathbf{K}_{\mathbf{p}}$ then

23: Allocate HeNB $i$ to the partitioned spectrum.

24: else

25: Allocate HeNB $i$ to the full spectrum.

26: end if

Note that the centralized solution needs the crosstier feedback for all femtocell links through the backhaul network. However, collecting all the channel information requires a significant amount of control overhead, which makes obtaining the solution very hard in practice. The centralized scheme also has the scalability problem since its computational complexity increases with the square of the network size. To overcome the implementation problems in the centralized scheme, we develop a decentralized algorithm next, which runs with low cross-tier control overhead. 


\section{Decentralized spectrum allocation and partitioning schemes}

In this section, we develop a decentralized spectrum allocation and partitioning scheme. In our solution, each HeNB determines whether it will use full spectrum or partitioned spectrum with a minimal cross-tier feedback.

\subsection{Decentralized algorithm}

The decentralized spectrum allocation algorithm also needs SIR measurement tests at HUE and HeNB. We describe a decentralized spectrum allocation scheme in Algorithm 2, and Figure 3 presents the control signalling procedures.

$i$ In the first test, MeNB transmits a pilot signal with beamforming transmission. If HUE $i$ can achieve the required SIR $\gamma_{f}^{q}$, it notifies its associated HeNB $i$ that it belongs to $\mathbf{F}_{1}$, otherwise $\mathbf{F}_{2}$.

$i i$ In the second test, MUE broadcasts a pilot signal with omnidirectional transmission, and HeNB $i$ reversely estimates its interference to MUE from the received signal strength, assuming the symmetric channel property. HeNB $i$ transmits its cross-tier interference to MUE, $I_{i}$.

iii MUE measures the cross-tier interference $S_{\mathrm{F}_{1}}$ and informs the HeNBs in $F_{1}$ of $S_{\mathrm{F}_{1}}$. The cross-tier feedback from MUE to HeNBs varies depending on the HeNB selection policies that will be described in the following section; they are the equal selection policy and the interference weighted selection policy. With the equal selection policy, MUE need to inform all HeNBs in $\mathbf{F}_{\mathbf{1}}$ of $S_{\mathrm{F}_{1}}$ through the backhaul network. However, in the case of the interference weighted selection policy, MUE transmits a pilot signal with controlled power $P_{S}:=P_{t} S_{\mathrm{F}_{1}}$. HeNBs recover $S_{\mathrm{F}_{1}}$ from the received signal of $P_{S}$, because each HeNB $i$ is already aware of the channel response $h_{i}$ between MUE and HeNB $i$ through the former cross-tier handshake to estimate $I_{i}$ in $i$.

$i v$ Each HeNB $i$ decides to use the full spectrum with the probability $p_{s}$, and otherwise, the partitioned spectrum. To avoid collecting all the channel status at MUE, which is a big burden, each HeNB applies a probabilistic decision mechanism. In the following Section 4.2, we explain how to calculate the probability $p_{s}$ using $S_{\mathrm{F}_{1}}$.

\section{Algorithm 2. Decentralized spectrum allocation scheme}

1: $i$ MeNB transmits a pilot with beamforming transmission.

2: for each HUE $i$ do

$$
\begin{array}{ll}
\text { 3: } & \text { if } \gamma_{f_{i}}>\gamma_{f}^{q} \text { then } \\
\text { 4: } & \text { HeNB } i \rightarrow \mathbf{F}_{\mathbf{1}} \\
\text { 5: else } & \\
\text { 6: } & \text { HeNB } i \rightarrow \mathbf{F}_{2} \\
\text { 7: } & \text { end if }
\end{array}
$$

8: end for

9: HeNB $i$ determines whether to belong to $\mathbf{F}_{\mathbf{1}}$ according to the HUE $i$ 's feedback.

10: ii MUE transmits a pilot signal omnidirectionally.

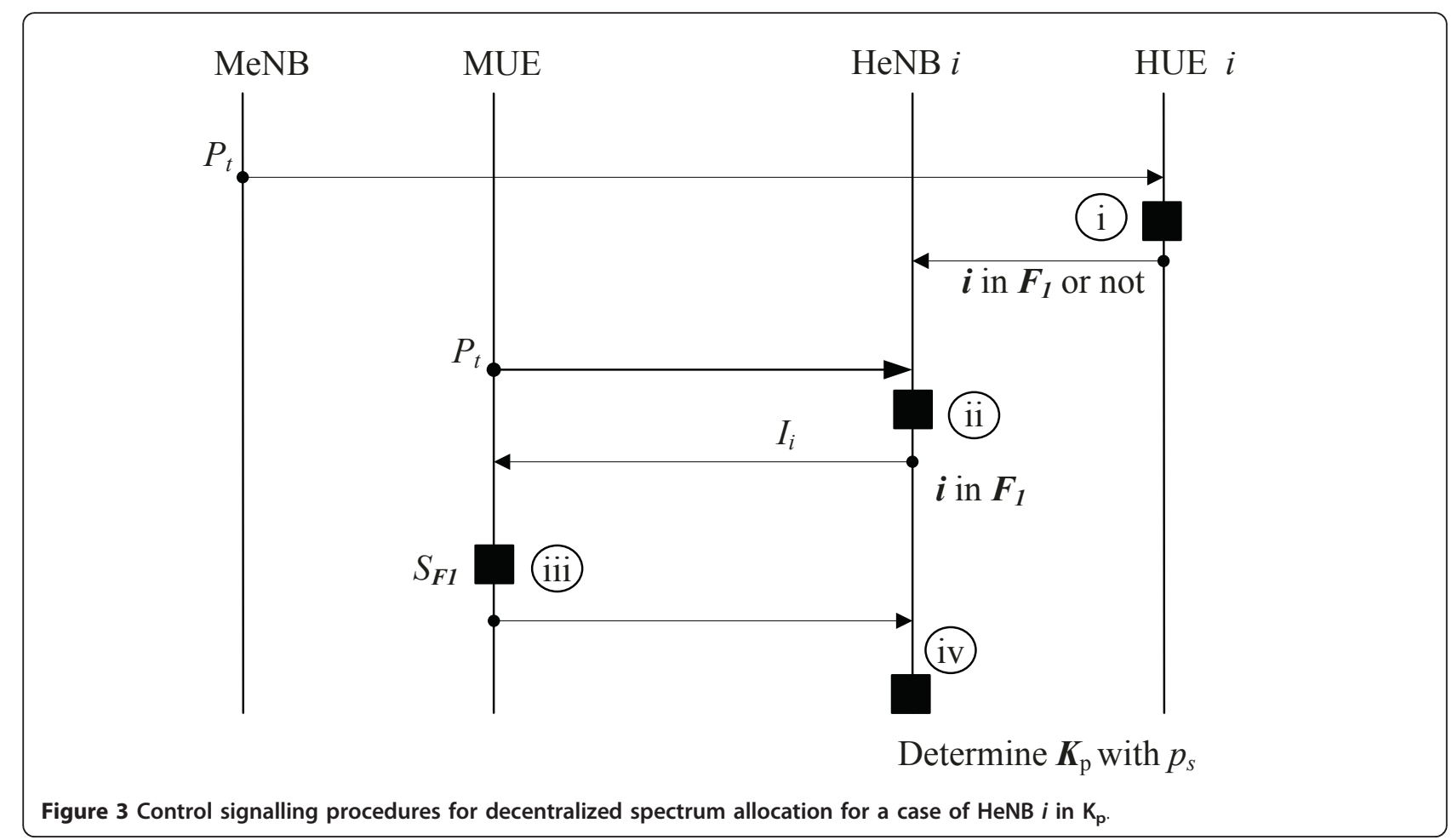


11: for each HeNB $i$ in $\mathbf{F}_{\mathbf{1}}$ do

12: HeNB $i$ measures the received signal strength for the MUE's pilot.

13: $\quad$ HeNB $i$ generates $I_{i}$ to MUE.

14: end for

15: iii MUE measures the cross-tier interference $S_{\mathrm{F}_{1}}$.

16: MUE informs the HeNBs in $F_{1}$ of the $S_{\mathbf{F}_{1}}$.

17: $i v$ for each HeNB $i$ in $\mathbf{F}_{\mathbf{1}}$ do

18: Define the sharing probability $p_{s}$ by using $S_{\mathrm{F}_{1}}$.

19: Allocate HeNB $i$ to $\mathbf{K}_{\mathbf{s}}$ with the probability $p_{s}$.

20: Otherwise, allocate $\mathrm{HeNB} i$ to $\mathbf{K}_{\mathbf{p}}$.

21: end for

We now determine $v_{p}$ with the decentralized spectrum partitioning scheme which details are provided in Algorithm 3, and Figure 4 presents the control signalling procedures.

Algorithm 3. Decentralized spectrum partitioning scheme 1: $i$ for each HeNB $i$ in $\mathbf{K}_{\mathbf{p}}$ do

2: $\quad$ Transmits a pilot signal with controlled power $P_{c o}^{i}$.

3: end for

4: ii MUE measures $I_{c o}$ and determines $\mathbf{K}_{\mathbf{p}}$ and $v_{p}$.

5: MUE transmits a pilot signal with controlled power

$P_{v}$ to inform each HeNB $i$ in $\mathbf{K}_{\mathbf{p}}$ of the $v_{p}$.

6: if HeNB $i \in \mathbf{K}_{\mathbf{p}}$ then

7: $\quad$ Allocate HeNB $i$ to the partitioned spectrum. 8: else

9: Allocate HeNB $i$ to the full spectrum.

10: end if

$i$ Each HeNB $i$ in $\mathbf{K}_{\mathbf{p}}$ transmits a pilot signal with controlled power $P_{c o}^{i}$ simultaneously, where $P_{c o}^{i}=\frac{P_{t}}{h_{i}}$ and $P_{t}$ denotes the transmission power of the pilot signal, which is predefined.

ii MUE measures the received interference from the HeNBs in $\mathbf{K}_{\mathbf{p}}$, denoted by $I_{c o}$, which is given as

$$
I_{c o}=\sum_{i \in \mathbf{K}_{\mathbf{P}}} h_{i} P_{c o}^{i}=\sum_{i \in \mathbf{K}_{\mathbf{P}}} h_{i} \frac{P_{t}}{h_{i}}=P_{t}\left|\mathbf{K}_{\mathbf{p}}\right| .
$$

Then MUE estimates $\left|\mathbf{K}_{\mathbf{p}}\right|$ by the following,

$$
\left|\mathbf{K}_{\mathbf{p}}\right|=\frac{I_{c o}}{P_{t}} .
$$

MUE calculates $v_{p}$ using $\left|\mathbf{K}_{\mathbf{p}}\right|$ and Equation (7), and sends it to MeNB. MeNB uses the allocated spectrum for itself. To inform the HeNBs in $\mathbf{K}_{\mathbf{p}}$ of the $v_{p}$, MUE transmits a pilot signal with controlled power $P_{v}:=P_{t} v_{p}$. HeNB $i$ in $\mathbf{K}_{\mathbf{p}}$ recovers $v_{p}$ from the received signal of $P_{v}$ and uses the partitioned spectrum, while other HeNBs uses the full spectrum.

Unlike the centralized control, which requires a large amount of channel feedback information via the backhaul network and incurs unacceptable control overhead and delay, our decentralized solution suffices with a small amount of cross-tier feedback information owing to the use of aggregated interference information.

\subsection{Probabilistic selection policies and outage probability} In our decentralized solution, we mitigate interference by allowing each HeNB to determine by itself whether to use the full or partitioned spectrum. In this section, we consider two types of spectrum sharing probability; one is the equal selection probability $p_{s}^{E}$, and the other the interference weighted selection probability $p_{s}^{W}$. Then we compare their performance in terms of outage probability.

Similar to the centralized scheme, the decentralized allocation scheme categorizes HeNBs into two groups: $\mathbf{F}_{\mathbf{1}}$ and $\mathbf{F}_{\mathbf{2}}$ according to the measured SIR at HUE. Then each HeNB belonging to $\mathbf{F}_{\mathbf{1}}$ determines whether to use the full spectrum with the probability $p_{s}$.

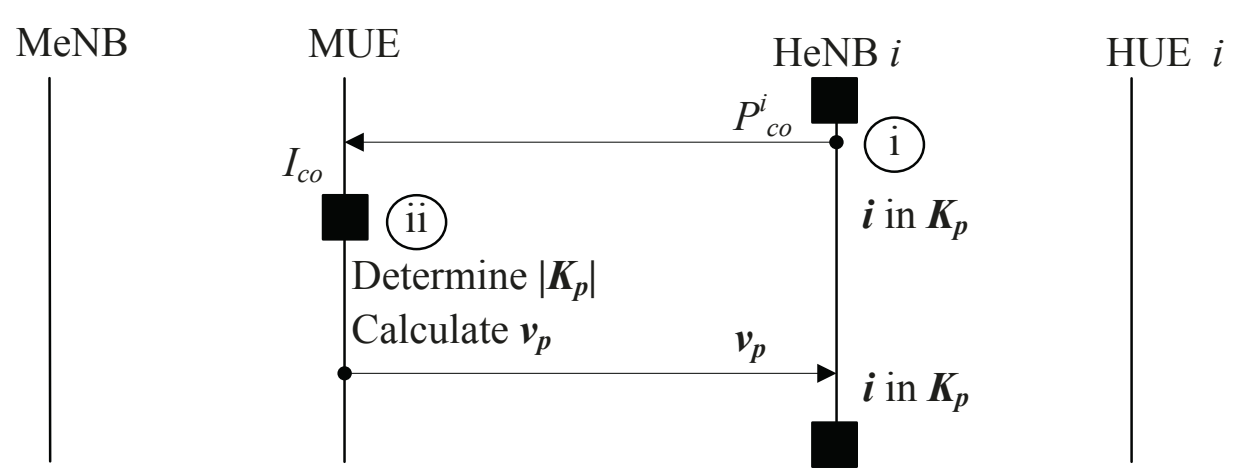

Figure 4 Control signalling procedures for decentralized spectrum partitioning for a case of HeNB $i$ in $K_{p}$. 


\subsubsection{Equal selection policy}

Since MUE suffers from the aggregate interference $S_{\mathbf{F}_{1}}$, i.e., $\sum_{i \in \mathrm{F}_{1}} I_{i}, S_{\mathrm{F}_{1}}$ should be smaller than the requirement $S_{m}^{q}:=\frac{P^{R}}{\gamma_{m}^{q}}$. If not, some HeNBs in $\mathbf{F}_{\mathbf{1}}$ should be allocated for the partitioned spectrum. This means that the other HeNBs are able to use the full spectrum.

In the equal selection policy, we define the sharing probability $p_{s}^{E}$ as

$$
p_{s}^{E}=\min \left\{1, \frac{S_{m}^{q}}{S_{\mathrm{F}_{1}}}\right\}
$$

Because this policy gives the spectrum sharing opportunity equally to all $\mathrm{HeNBs}$ in $\mathbf{F}_{\mathbf{1}}$, the resultant crosstier interference at MUE with the equal selection policy is given by

$$
S_{m}:=p_{s}^{E} \sum_{i \in \mathbf{F}_{1}} I_{i}
$$

Thus the expectation of $S_{m}$ is less than or equal to $S_{m}^{q}$. However, as this selection policy operates in a probabilistic way, we cannot guarantee that $S_{m}$ is smaller than $S_{m}^{q}$. If the spectrum sharing policy cannot satisfy the MUE's QoS requirement, an outage occurs. Assuming that the aggregate interference follows a normal distribution (see Equation (18) in the Appendix), we calculate the outage probability according to Q-function as

$$
p_{o}^{j}\left(p_{s}^{E}, \gamma_{m}^{q}\right)=Q\left(\frac{\Psi_{j} S_{m}^{q}-n_{F}^{j} p_{s}^{E} \xi}{\sqrt{n_{F}^{j}} p_{s}^{E} \varphi}\right)
$$

where we assume that $I_{i}$ 's are random variables with the mean $\xi$ and variance $\phi^{2}$ with omnidirectional transmission. Let $\Psi_{j}$ denote the average beamforming gain when MeNB or HeNBs transmit with the $j$ th beamforming transmission. With the beamforming gain $\Psi_{j}$, the number of HeNBs in $\mathbf{F}_{\mathbf{1}}$ is denoted by $n_{F}^{j}$, i.e., $n_{F}^{j}=\left|\mathbf{F}_{1}^{\mathbf{j}}\right|$. Refer to Appendix 1.1 for detailed derivation of (12).

Since $Q$-function is a monotonically decreasing function, the outage probability lowers with a larger beamforming gain $\Psi_{j}$ and a smaller mean $\xi$ and variance $\phi^{2}$. The outage probability increases with a smaller $S_{m}^{q}$, i.e., stricter SIR requirement of $\gamma_{m}^{q}$. However, the smaller $S_{m}^{q}$ also reduces $n_{F}^{j}$ and $p_{s}^{E}$. Accordingly the combination of these affects the resultant outage probability.

\subsubsection{Interference weighted selection policy}

We can increase $\left|\mathbf{K}_{\mathbf{s}}\right|$ by allocating the HeNBs that generate severe cross-tier interference to the set $\mathbf{K}_{\mathbf{p}}$. However, as noted in our centralized scheme, it causes inevitable delay in determining which HeNB should belong to the partitioned set first due to the cross-tier feedback. To this end, we develop an interference weighted selection policy, where each HeNB determines whether to use the full spectrum according to the sharing probability $p_{s}^{W}$, which is given by

$$
p_{s}^{W}(i)=\min \left\{1, \frac{S_{m}^{q}}{n_{F} I_{i}}\right\}
$$

Under this policy, HeNBs that generate moderate interference to MUE, smaller than $\frac{S_{m}^{q}}{n_{F}}$, use the full spectrum, otherwise, HeNB $i$ belongs to $\mathbf{K}_{\mathbf{p}}$ with the probability of $\frac{S_{m}^{q}}{n_{F} I_{i}}$. Then we obtain the outage probability of MUE as

$$
p_{o}^{j}\left(p_{s}^{W}, \gamma_{m}^{q}\right)=Q\left(\frac{\Psi_{j} S_{m}^{q}-n_{S}^{j} \xi-\Psi_{j} S_{m}^{q} \frac{n_{L}^{j}}{n_{F}^{j}}}{\sqrt{n_{S}^{j}} \varphi}\right)
$$

Refer to Appendix 1.2 for detailed derivation of (14). Under the interference weighted selection policy, the outage probability lowers with a larger beamforming gain $\Psi_{j}$ and a smaller mean $\xi$ and variance $\phi^{2}$ of the cross-tier interference $I_{i}$. For a given outage constraint, with a larger beamforming gain, a larger number of HeNBs use the full spectrum, resulting in enhanced cell capacity.

In summary, we have developed a practical decentralized solution for spectrum allocation which does not require individual channel information for all the cross-tier links, but it simply requires aggregated interference information. The interference weighted selection policy achieves lower outage probability and higher spectrum efficiency than the equal selection policy. Note that the centralized scheme, though impractical, does not experience an outage at all because it uses all the co-tier and cross-tier channel information in making a decision.

\section{Performance evaluation}

In this section, the spectrum efficiency is evaluated through simulations considering the aver-age number of HeNBs which use the full spectrum. The centralized and decentralized scheme are compared with a scheme without spectrum partitioning in terms of outage 
probability. Further, we investigate how the shared spectrum ratio affects cell capacity and utility.

In our simulations, we consider a macrocell site with 100 femtocells, i.e., $\left|\mathbf{K}_{\mathbf{t}}\right|=100$. We assume that MeNB is located at the center and its transmission range $R_{m}$ is $500 \mathrm{~m}$. Each HeNB is randomly located within the macrocell site and its transmission range $R_{f}$ is $20 \mathrm{~m}$. We repeatedly simulated over different random topologies and provide their average results. We set the utility weights for MUE and HUE at $w_{m}=10$ and $w_{f}=1$, respectively.

MeNB and HeNBs are configured with three different numbers of beams $N_{b} \in\{1,4,8\}$, which represent the beamforming sharpness of the main lobe (i.e., $\theta_{m}=\frac{2 \pi}{N_{b}}$ ). The beam gains of the main lobe and the side lobe are denoted by $g_{m}$ and $g_{s}$, respectively, in $\mathrm{dB}$ scale and the average beamforming gain in the twotier network by $\Psi\left(N_{b}\right)$. The path loss exponent parameters $\alpha$ 's for MUE and HUEs are uniformly distributed in $[3,5]^{\mathrm{a}}$. We set the UE noise figure at $-174 \mathrm{dBm} / \mathrm{Hz}$ and the spectrum bandwidth at $20 \mathrm{MHz}$ which follow the 3GPP LTE specifications. The system parameters and notations are summarized in Table 3.

Table 3 Definition of notations

\begin{tabular}{|c|c|}
\hline Symbol & Description \\
\hline$R_{m}$ & Macrocell transmission radius \\
\hline$R_{f}$ & Femtocell transmission radius \\
\hline$P^{R}$ & Desired received signal strength at UE \\
\hline$\alpha$ & Path loss exponent \\
\hline $\mathrm{K}_{\mathrm{t}}$ & Set of femtocells \\
\hline $\mathrm{K}_{\mathrm{s}}$ & Set of femtocells with shared spectrum \\
\hline $\mathrm{K}_{\mathrm{p}}$ & Set of femtocells with partitioned spectrum \\
\hline$v_{s}$ & Ratio of shared spectrum \\
\hline $\begin{array}{c}v_{p}=(1- \\
\left.v_{s}\right)\end{array}$ & Ratio of partitioned spectrum \\
\hline$N_{b}$ & Number of beams \\
\hline$g_{m}$ & Beamforming gain for the main lobe \\
\hline$g_{s}$ & Beamforming gain for the side lobe \\
\hline$\gamma_{m}$ & Measured SIR at MUE \\
\hline$\gamma_{f}$ & Measured SIR at HUE \\
\hline$\gamma_{m}^{q}$ & Required SIR at MUE \\
\hline$\gamma_{f}^{q}$ & Required SIR at HUE \\
\hline $\mathrm{F}_{1}$ & $\begin{array}{l}\text { Set of HeNBs whose associated HUE has a SIR greater than } \\
\qquad \gamma_{f}^{q}\end{array}$ \\
\hline$S_{\mathrm{F}_{1}}$ & Interference at MUE from HeNBs in $\mathbf{F}_{\mathbf{1}}$ \\
\hline$S_{m}$ & Interference at MUE with the HeNB selection policy \\
\hline$S_{m}^{q}$ & Permitted interference at MUE for $\gamma_{m}^{q}$ \\
\hline$p_{s}$ & Probability to use full spectrum \\
\hline$p_{s}^{E}$ & $p_{s}$ of the equal selection policy \\
\hline$p_{s}^{W}$ & $p_{s}$ of the interference weighted selection policy \\
\hline
\end{tabular}

At first, we compare the centralized and decentralized spectrum allocation scheme with the distance based allocation scheme which classifies femtocells into inner and outer types according to the distance from MeNB. Inner femtocells use partitioned spectrum while outer femtocells use shared spectrum. Because existing hybrid spectrum schemes have not con sidered beamforming environments, we use the distance based scheme with beamforming in [23].

The average number $E\left[\left|\mathbf{K}_{\mathbf{s}}\right|\right]$ of HeNBs which use the full spectrum and the CDF (cumulative distribution function) of $\left|\mathbf{K}_{\mathbf{s}}\right|$ when $\gamma_{m}^{q}=0 \mathrm{~dB}$ are shown in Figures 5 and 6 , respectively. In general, sharper beamforming leads a larger number of HeNBs to share the spectrum with the macrocell network, $\left|\mathbf{K}_{\mathbf{s}}\right|$. For a given beamforming gain, the centralized algorithm, marked as 'Centralized', has the highest $\left|\mathbf{K}_{\mathbf{s}}\right|$. The $\left|\mathbf{K}_{\mathbf{s}}\right|$ of the decentralized schemes which are the equal selection policy, marked as 'Decentralized Equal', and the interference weighted selection policy, marked as 'Decentralized Weight' are is nearly comparable to that in the centralized one. However, the distance based scheme, represented as 'Distance-based', has a lower number of $E\left[\left|\mathbf{K}_{\mathbf{s}}\right|\right]$ because it operates with a fixed distance threshold, following the average channel model without actual channel state information.

To suppress interference, the interference cancelation with MIMO and beamforming transmission techniques are used. However, even with beamforming transmission, the cross-tier interference where all HeNBs share the macrocell spectrum without interference mitigation still remains severe and degrades the outage performance.

The outage performance of MUE with and without cross-tier interference mitigation is presented in Figure 7. We represent the cochannel deployment with beamforming transmission, where all $\mathrm{HeNBs}$ share the macrocell spectrum without interference mitigation, marked as 'Without IM'. Without spectrum partitioning scheme, MUE suffers from severe crosstier interference and the outage probability goes up to the range $\left[10^{-1}\right.$, $10^{-2}$ ]. Our decentralized allocation and partitioning schemes, particularly the interference weighted policy, reduces the outage probability successfully by allocating severe interference generating HeNBs to the partitioned spectrum with high probability. It also shows that the outage probability lowers with the beamforming gain. Note that the centralized scheme does not experience an outage at all owing to the use of all the co-tier and cross-tier channel information, and the distance based scheme does not experience an outage either because of its conservative spectrum sharing policy, i.e., lower number of $\left|\mathbf{K}_{\mathbf{s}}\right|$. 


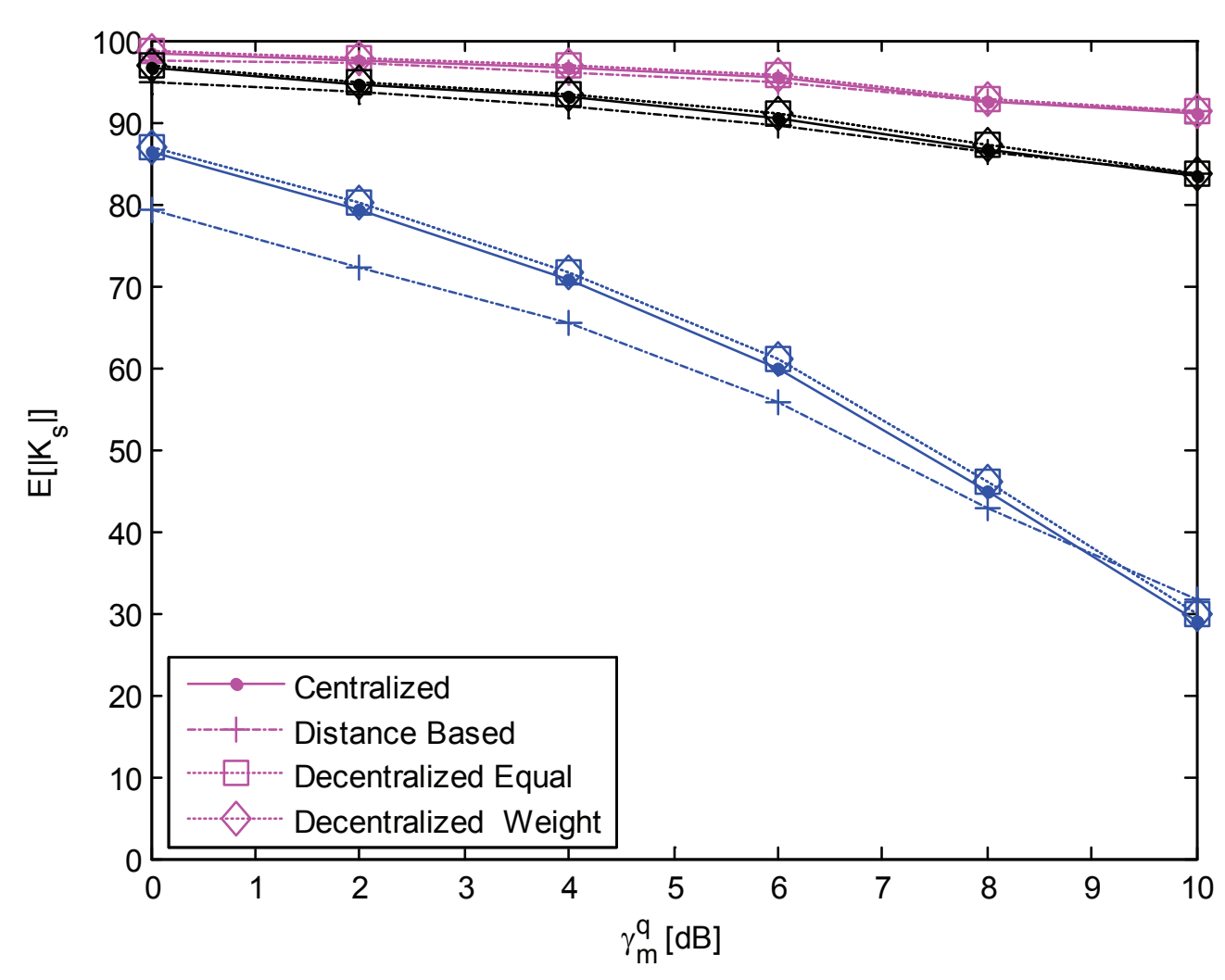

Figure 5 Average number of sharing HeNBs versus required SIR at MUE.

Figure 8 summarizes the trend of outage probability and $E\left[\left|\mathbf{K}_{\mathbf{s}}\right|\right]$ according to different SIR requirements. In the 'Without IM' scheme, all HeNBs use the cochannel with macrocell network inducing the severe cross-tier interference. The outage probability of 'Without IM' is around $10^{-1}$ when $N_{b}=4$, and reduces to $10^{-2}$ when $N_{b}$ $=8$. It shows that the interference mitigation significantly improves the outage probability, especially when the decentralized weight policy is applied. In general, with a stricter SIR requirement, the outage occurs more frequently. However, $E\left[\left|\mathbf{K}_{\mathbf{s}}\right|\right]$ in the proposed algorithm decreases with higher $\gamma_{m}^{q}$ as shown in Figure 5, the outage probability gets lowered. As a result, we notice that the outage probability decreases moderately and then increases with the increase of $E\left[\left|\mathbf{K}_{\mathbf{s}}\right|\right]$, i.e., the decrease of $\gamma_{m}^{q}$.

The cell capacity and utility performance according to the requirement SIR at MUE $\gamma_{m}^{q}$ with $N_{b}=1$ are shown in Figures 9 and 10, respectively. The spectrum partitioning scheme with the fixed ratios of $v_{s}=0.5$ and 0.9 is compared with our decentralized spectrum partitioning scheme. With the increase of $\gamma_{m}^{q}$, the capacity of each UE increases on a logarithmic scale, but $\left|\mathbf{K}_{\mathbf{s}}\right|$ decreases as shown in Figure 5 . Therefore, the cell capacity increase moderately with $\gamma_{m}^{q}$. The centralized scheme achieves the highest cell capacity and utility performance, and our probabilistic spectrum allocation and partitioning schemes are comparable to the centralized one. However, the fixed spectrum partitioning scheme with the ratios of $v_{s}=0.5$ and 0.9 has lower cell capacity and utility compared to our schemes. The 'Without IM' scheme shows the lowest cell capacity and utility because of severe cross-tier interference.

\section{Conclusion}

In this article, we proposed spectrum allocation and partitioning algorithms to mitigate cross-tier interference in downlink under beamforming environments. We analytically derived the optimal ratio of spectrum partitioning to maximize the cell utility considering both efficiency and fairness. Because our decentralized scheme uses aggregated cross-tier interference in a probabilistic manner, it requires less cross-tier feedback. Our simulation results show that the proposed decentralized algorithm with the interference weighted HeNB selection policy is comparable to the centralized scheme in terms of the total cell capacity and utility. Owing to the use of low cross-tier control overhead, it also effectively solves the 


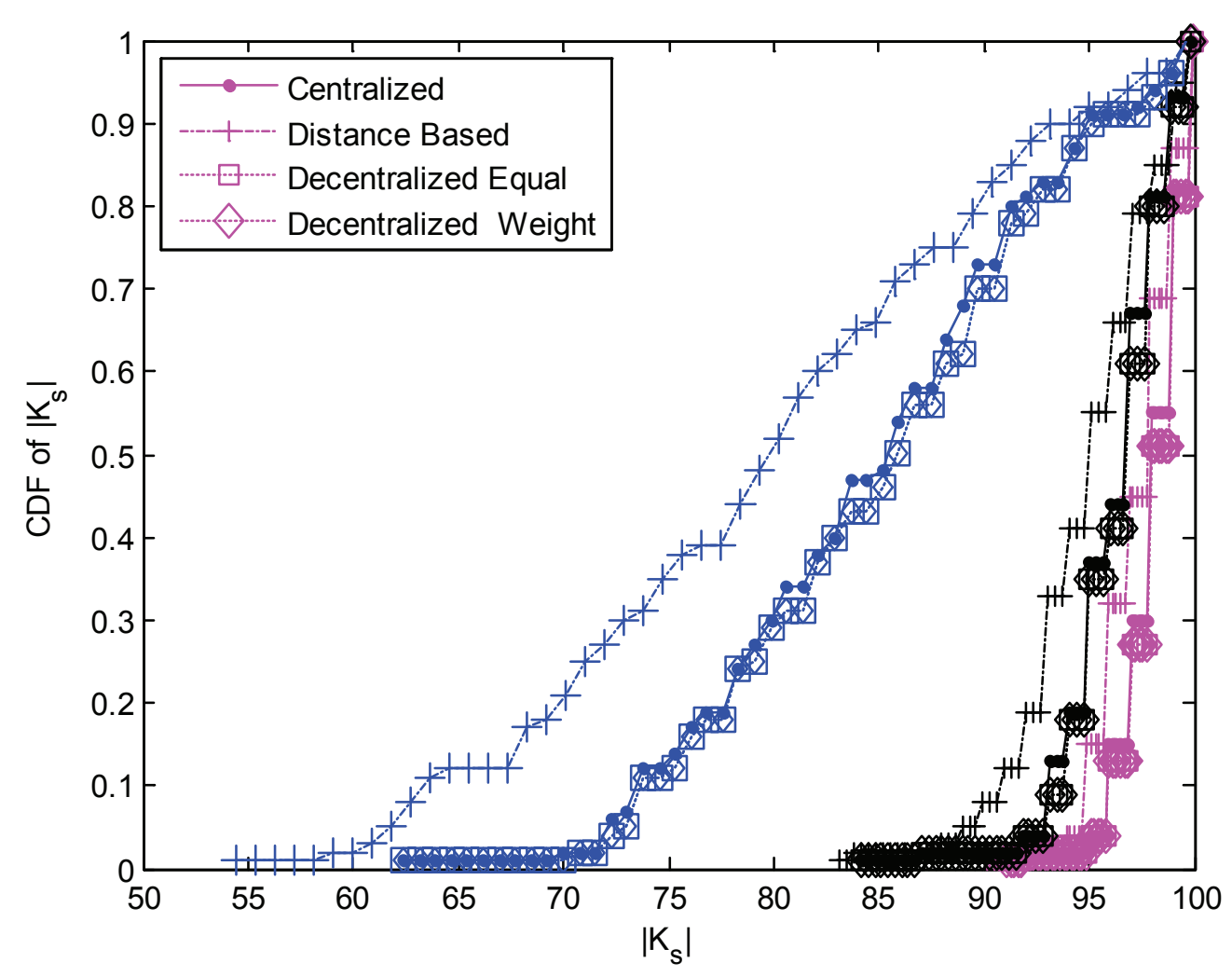

Figure $6 \mathrm{CDF}$ of $\left|\mathrm{K}_{\mathrm{s}}\right|$ when the required SIR at MUE is $0 \mathrm{~dB}$.

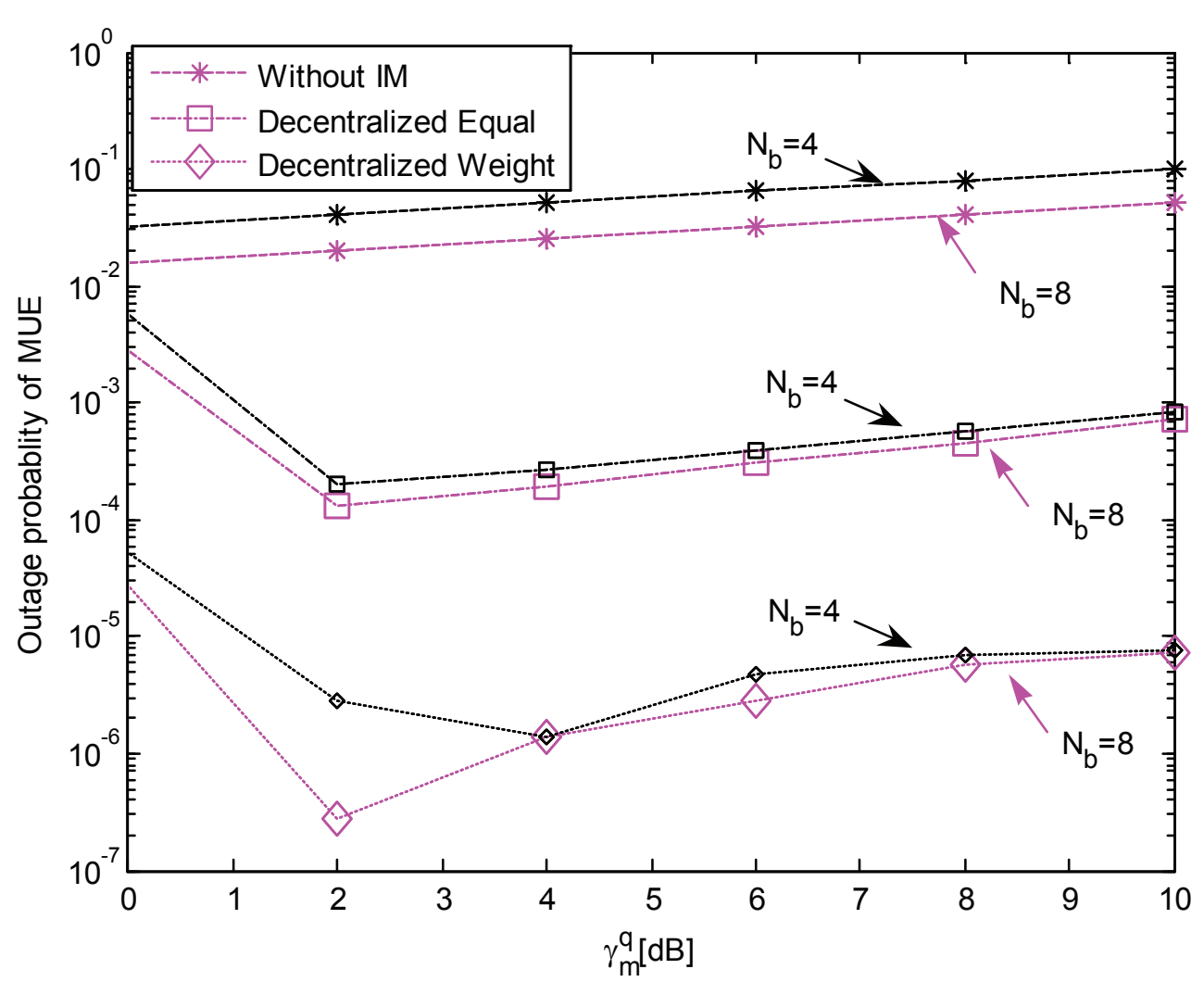

Figure 7 Outage probability of MUE with and without the interference mitigation versus required SIR at MUE. 


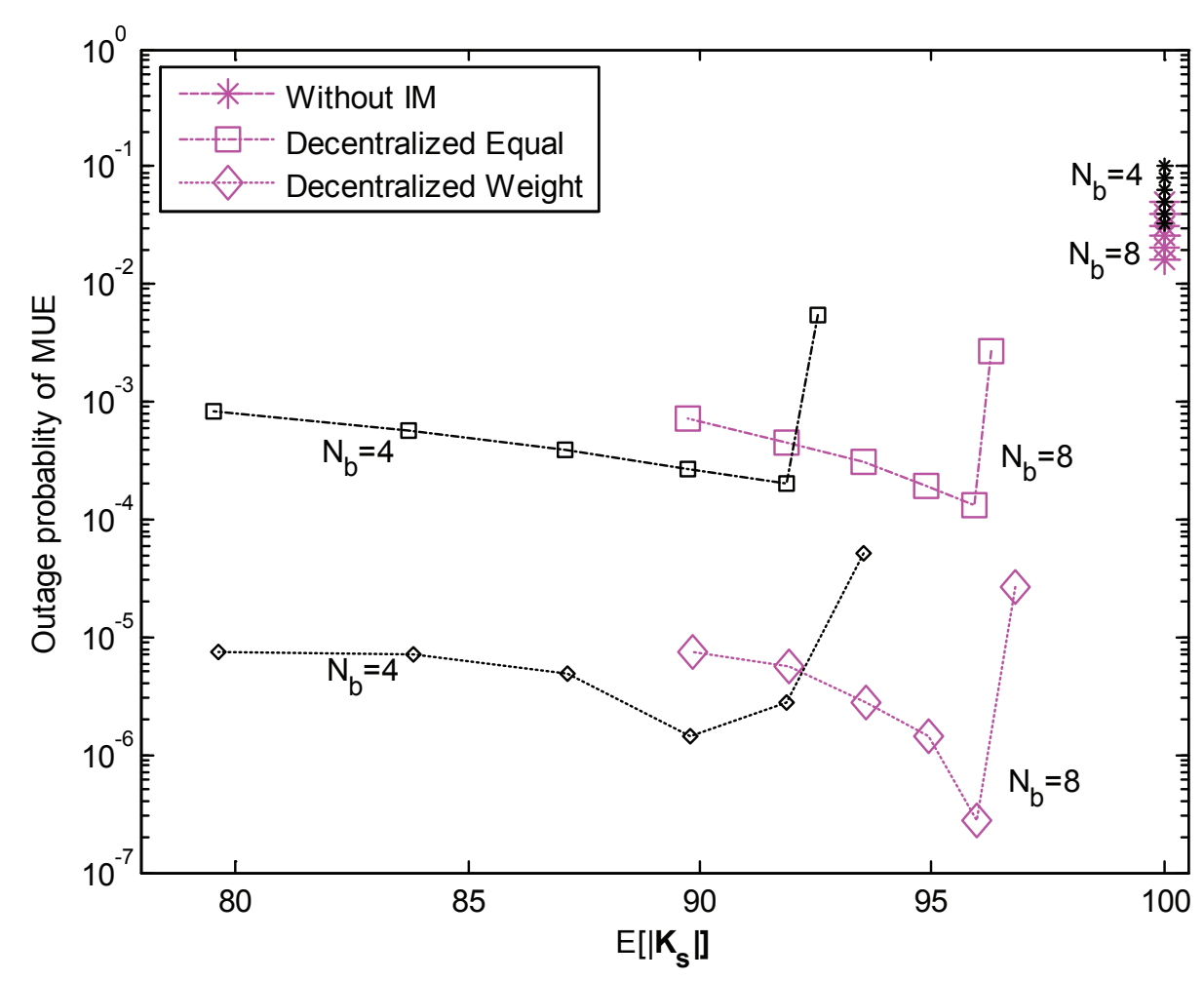

Figure 8 Outage probability of MUE versus average number of $K_{s}$.

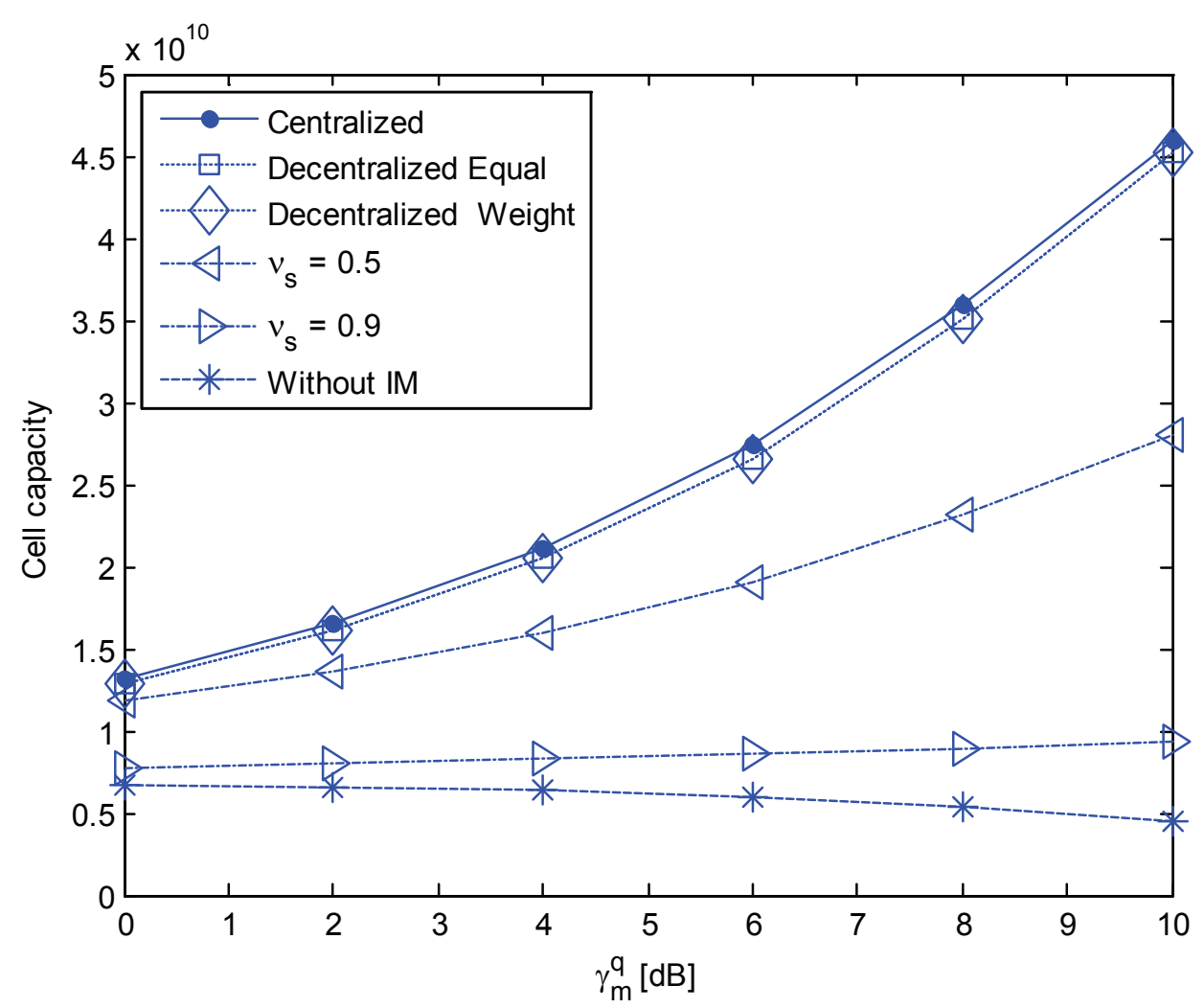

Figure 9 Cell capacity versus required SIR at MUE with $N_{b}=1$. 


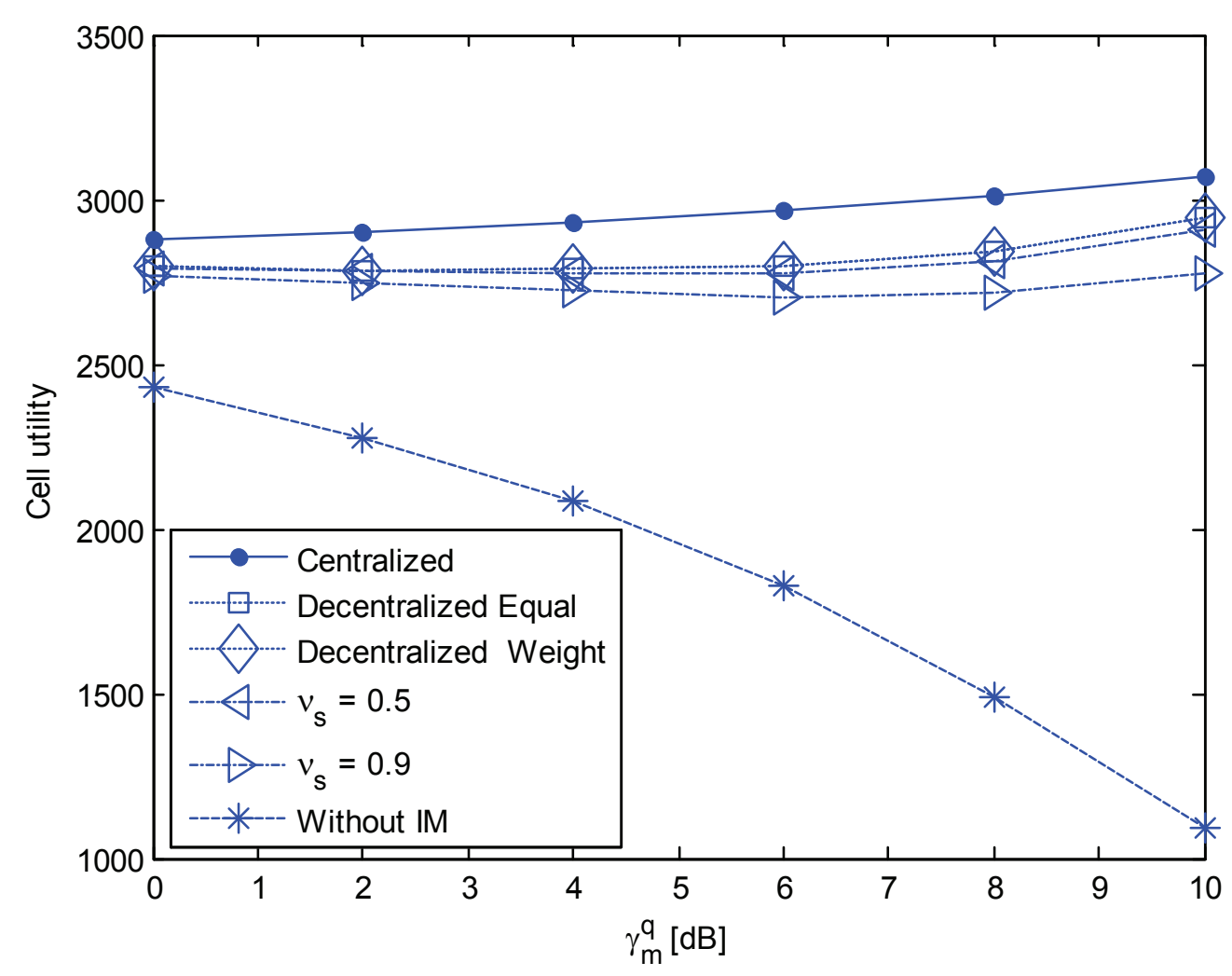

Figure 10 Cell utility versus required SIR at MUE with $N_{b}=1$.

cross-tier interference problem in a large scale two-tier network.

\section{Appendix 1: derivation of outage probability of the proposed selection policies}

According to the central limit theorem, the aggregate interference at MUE, $S_{m}$, approaches a normal distribution when each $I_{i}$ are independent identically distributed regardless of the shape of each distribution of individual $I_{i}$.

The outage occurs when $S_{m}$ is larger than the limited interference $S_{m}^{q}$. The outage probability, i.e., transmission failure probability due to the cross-tier interference is

$$
\begin{aligned}
p_{o}\left(p_{s}, \gamma_{m}^{q}\right) & =\operatorname{Pr}\left(S_{m} \geq S_{m}^{q}\right) \\
& =1-F\left(S_{m}=S_{m}^{q} ; \mu, \sigma^{2}\right),
\end{aligned}
$$

where the distribution of $S_{m}$ is approximately normal with the mean $\mu$ and variance $\sigma^{2}$, i.e., $N\left(\mu, \sigma^{2}\right)$. Let $f\left(S_{m}\right.$; $\left.\mu, \sigma^{2}\right)$ and $F\left(S_{m} ; \mu, \sigma^{2}\right)$ denote the PDF and CDF of $S_{m}$, respectively.

Using Q-function, we obtain the outage probability of MUE as

$$
p_{o}\left(p_{s}, \gamma_{m}^{q}\right)=Q\left(\frac{S_{m}^{q}-\mu}{\sigma}\right)
$$

Next, we derive the outage probability by finding the mean $\mu$ and variance $\sigma^{2}$ of $S_{m}$ for the equal and interference weighted selection policies.

\section{Appendix 1.1: Equal selection policy}

We apply the equal election policy to mitigate cross-tier interference given in Equation (10). If $S_{\mathrm{F}_{1}}<S_{m}^{q}$, we do not need to apply the equal selection policy. All the HeNBs in $\mathbf{F}_{\mathbf{1}}$ which already satisfy the SIR requirement at HUEs share the licensed spectrum with the macrocell, i.e., $p_{s}^{E}=1$. Otherwise, i.e., $S_{\mathrm{F}_{1}} \geq S_{m}^{q}$, HeNBs in $\mathbf{F}_{\mathbf{1}}$ share the macrocell spectrum with the probability of $p_{s}^{E}$.

When the equal selection with $p_{s}^{E}$ is applied to meet the MUE's SIR requirement, the cross-tier interference at MUE with beamforming gain $\Psi_{j}$ is given by

$$
S_{m}^{j}\left(p_{s}^{E}, \gamma_{m}^{q}\right)=p_{s}^{E} \sum_{i \in \mathrm{F}_{1}^{\mathrm{j}}} I_{i j} .
$$


Let $I_{i}$ denotes the cross-tier interference to MUE by HeNB $i$ 's omnidirectional transmission. We assume $I_{i}^{\prime}$ s distribution has mean $\xi$ and variance $\phi^{2}$. Considering the $j$ th beamforming transmission, $I_{i j}$ denotes the crosstier interference to MUE by HeNB $i$ 's beamforming transmission with gain $\Psi_{j}$. Then the distribution of $I_{i j}$ has the mean of $\xi / \Psi_{j}$ and variance of $\left(\phi / \Psi_{j}\right)^{2}$. The mean and variance of $S_{m}^{j}$ with the equal selection policy is scaled by $n_{F}^{j} p_{s}^{E}$ and $n_{F}^{j}\left(p_{s}^{E}\right)^{2}$, respectively, where $n_{F}^{j}$ is the number of HeNBs in $\mathbf{F}_{1}^{\mathbf{j}}$, i.e., $\left|\mathbf{F}_{1}^{\mathbf{j}}\right|$.

Therefore, the distribution of the cross-tier interference at MUE with the equal selection policy is

$$
S_{m}^{j}\left(p_{s}^{E}, \gamma_{m}^{q}\right) \rightarrow N\left(\frac{n_{F}^{j} p_{s}^{E}}{\Psi_{j}} \xi, \frac{n_{F}^{j} p_{s}^{E^{2}}}{\Psi_{j}^{2}} \varphi^{2}\right) .
$$

From Equation (16), we obtain the outage probability of MUE as

$$
p_{o}^{j}\left(p_{s}^{E}, \gamma_{m}^{q}\right)=Q\left(\frac{\Psi_{j} S_{m}^{q}-n_{F}^{j} p_{s}^{E} \xi}{\sqrt{n_{F}^{j}} p_{s}^{E} \varphi}\right) .
$$

\section{Appendix 1.2: Interference weighted selection policy}

When we apply the interference weighted selection policy to mitigate cross-tier interference, HeNBs which generate smaller cross-tier interference than $\frac{S_{m}^{q}}{n_{F}}$ use the full spectrum. Other wise, the other HeNBs are assigned to the partitioned set with the probability $\frac{S_{m}^{q}}{n_{F} I_{i}}$ as defined in Equation (13). With the interference weighted selection policy, we obtain the cross-tier interference at MUE as

$$
\begin{aligned}
S_{m}^{j}\left(p_{s}^{W}, \gamma_{m}^{q}\right) & =\sum_{i \in \mathbf{F}_{1}^{\mathrm{j}}} p_{s}^{W}(i) I_{i j} \\
& =\sum_{i \in \mathbf{S}} I_{i j}+\frac{S_{m}^{q}}{n_{F}} \sum_{i \in \mathbf{L}} \frac{I_{i j}}{I_{i j}},
\end{aligned}
$$

where $\mathrm{F}_{1}^{\mathrm{j}}$ denotes the set of HeNB $i$ where the SIR at HUE $i$ satisfies the requirement of $\gamma_{f}^{q}$ when the $j$ th beamforming is transmitted. $\mathbf{F}_{1}^{\mathrm{j}}$ is divided into two groups; they are $\mathbf{S}$ for the set of HeNBs that meet $I_{i}<S_{m}^{q} / n_{F}$, and $\mathbf{L}$, otherwise.

Denoting $n_{S}=|\mathbf{S}|$ and $n_{L}=|\mathbf{L}|$, i.e., $n_{F}=n_{S}+n_{L}$, we evaluate the upper bound of the average cross-tier interference at MUE as

$$
E\left[S_{m}\left(p_{s}^{W}(i), \gamma_{m}^{q}\right)\right]<n_{S} \frac{S_{m}^{q}}{n_{F}}+n_{L} \frac{S_{m}^{q}}{n_{F}}=S_{m}^{q}
$$

where $I_{i}<S_{m}^{q} / n_{F}$ for $\forall i \in \mathbf{S}$ and $\sum_{i \in \mathbf{S}} I_{i}<n_{S} \frac{S_{m}^{q}}{n_{F}}$ Thus the average cross-tier interference at MUE with the interference weighted selection policy is smaller than the limited interference level $S_{m}^{q}$.

From Equation (20), cross-interference at MUE consists of $n_{S}$ random variables, i.e., $\sum_{i \in \mathbf{S}} I_{i j}$ and the constant term, i.e., $n_{L}^{j} S_{m}^{q} / n_{F}^{j}$. The distribution of $S_{m}$ is a mean shift distribution of $\sum_{i \in \mathbf{S}} I_{i j}$, i.e., $N\left(\frac{n_{S}^{j}}{\Psi_{j}} \xi, \frac{n_{S}^{j}}{\Psi_{j}^{2}} \varphi^{2}\right)$ with $n_{L}^{j} S_{m}^{q} / n_{F}^{j}$ as below

$$
S_{m}\left(p_{s}^{W}, \gamma_{m}^{q}\right) \rightarrow N\left(\frac{n_{S}^{j}}{\Psi_{j}} \xi+n_{L}^{j} \frac{S_{m}^{q}}{n_{F}^{j}}, \frac{n_{S}^{j}}{\Psi_{j}^{2}} \varphi^{2}\right) .
$$

Therefore, we obtain the outage probability of MUE in the interference weighted selection policy as

$$
p_{o}^{j}\left(p_{s}^{W}, \gamma_{m}^{q}\right)=Q\left(\frac{\Psi_{j} S_{m}^{q}-n_{S}^{j} \xi-\Psi_{j} S_{m}^{q} \frac{n_{L}^{j}}{n_{F}^{j}}}{\sqrt{n_{S}^{j}} \varphi}\right) .
$$

\section{Endnote}

${ }^{a}$ In practical two-tier macro and femtocell systems, each user has different channel characteristics depending on indoor or outdoor environments. The channel modeling for a femtocell network is beyond the scope of our research. As our scheme uses limited information of channel feedback, it can be easily adapted to different channel modeling environments.

\section{Acknowledgements}

This study was supported in part by the NRF (National Research Foundation of Korea) grant funded by the Korea government (MEST) (No. 2011-0027517), in part by the MKE (The Ministry of Knowledge Economy), Korea, under the Convergence-ITRC (Convergence Information Technology Research Center) support program (NIPA-2012-H0401-12-1004) supervised by the NIPA (National IT Industry Promotion Agency) and in part by the research programs through National Research Foundation of Korea (NRF), funded by the Ministry of Education, Science, and Technology (No. 2011-0027517 and No. 2011-0008549).

\section{Author details}

${ }^{1}$ INMC, School of EECS, Seoul National University, Korea ${ }^{2}$ School of Electrical and Computer Engineering, UNIST, Ulsan, Korea

\section{Competing interests}

The authors declare that they have no competing interests. 
Received: 15 October 2011 Accepted: 3 May 2012

Published: 3 May 2012

\section{References}

1. V Chandrasekhar, J Andrews, A Gatherer, Femtocell networks: a survey. Communications Magazine, IEEE. 46(9), 59-67 (2008)

2. B Kim, J Kwon, J Lee, Utility-Based Subchannel Allocation for OFDMA Femtocell Networks, in Computer Communications and Networks (ICCCN) 2011 Proceedings of 20th International Conference on, IEEE, pp. 1-6 (2011)

3. D López-Pérez, A Valcarce, G De La Roche, J Zhang, OFDMA femtocells: a roadmap on interference avoidance. Communications Magazine, IEEE. 47(9), 41-48 (2009)

4. C Oh, M Chung, $\mathrm{H}$ Choo, $\mathrm{T}$ Lee, A novel frequency planning for femtocells in OFDMA-based cellular networks using fractional frequency reuse. Computational Science and Its Applications-ICCSA 2010. 6018, 96-106 (2010). doi:10.1007/978-3-642-12179-1_10

5. K Sundaresan, S Rangarajan, Efficient resource management in OFDMA femtocells, in Proceedings of the tenth ACM international symposium on Mobile ad hoc networking and computing, vol. 1. New Orleans, Louisiana, USA: ACM, pp. 33-42 (2009)

6. W Park, S Bahk, Resource management policies for fixed relays in cellular networks. Computer Communications. 32(4), 703-711 (2009). doi:10.1016/j. comcom.2008.11.039

7. C Chen, C Wang, S Chao, H Wei, DANCE: a game-theoretical femtocell channel exchange mechanism. ACM SIGMOBILE Mobile Computing and Communications Review. 14, 13-15 (2010)

8. V Chandrasekhar, J Andrews, Uplink capacity and interference avoidance for two-tier femtocell networks. Wireless Communications, IEEE Transactions on. 8(7), 3498-3509 (2009)

9. S Kishore, L Greenstein, H Poor, S Schwartz, Uplink user capacity in a multicell CDMA system with hotspot microcells. Wireless Communications, IEEE Transactions on. 5(6), 1333-1342 (2006)

10. C Kang, H Cho, D Sung, Capacity analysis of spectrally overlaid macro/ microcellular CDMA systems supporting multiple types of traffic. Vehicular Technology, IEEE Transactions on. 52(2), 333-346 (2003). doi:10.1109/ TVT.2002.807128

11. 3rd Generation Partnership Project (3GPP) TSGRAN, 3G Home NodeB Study Item Technical Report. 3GPP TR 25.820 V 8.1.0 (2008-05)

12. Y Kim, S Lee, D Hong, Performance analysis of two-tier femtocell networks with outage constraints. Wireless Communications, IEEE Transactions on 9(9), 2695-2700 (2010)

13. H Claussen, Performance of macro-and co-channel femtocells in a hierarchical cell structure, in Personal, Indoor and Mobile Radio Communications, 2007. PIMRC 2007. IEEE 18th International Symposium on, vol. 1. Athens, Greece: IEEE, pp. 1-5 (2007)

14. V Chandrasekhar, J Andrews, Spectrum allocation in tiered cellular networks, Communications, IEEE Transactions on. 57(10), 3059-3068 (2009)

15. S Park, W Seo, Y Kim, S Lim, D Hong, Beam subset selection strategy for interference reduction in two-tier femtocell networks. Wireless Communications, IEEE Transactions on. 9(11), 3440-3449 (2010)

16. Y Jeong, T Quek, H Shin, Beamforming Optimization for Multiuser Two-Tier Networks. Journal of Communication and Networks. 13, 327-338 (2011)

17. C You, Y Jung, S Cho, Beamforming Strategy Using Adaptive Beam Patterns and Power Control for Common Control Channel in Hierarchical Cell Structure Networks. Journal of Communication and Networks. 13, 319-326 (2011)

18. Y Bai, J Zhou, L Chen, Hybrid spectrum usage for overlaying LTE macrocell and femtocell, in Proceedings of the 28th IEEE conference on Global telecommunications, vol. 1. Honolulu, Hawaii, USA: IEEE Press, pp. 1642-1647 (2009)

19. M Erturk, H Aki, I Güvenc, H Arslan, Fair and QoS-Oriented Spectrum Splitting in Macrocell-Femtocell Networks, in GLOBECOM 2010, 2010 IEEE Global Telecommunications Conference, vol. 1. Miami, Florida, USA: IEEE, pp. 1-6 (2010)

20. I Guvenc, M Jeong, F Watanabe, H Inamura, A hybrid frequency assignment for femtocells and coverage area analysis for co-channel operation. Communications Letters, IEEE. 12(12), 880-882 (2008)

21. R Ramanathan, On the performance of ad hoc networks with beamforming antennas, in Proceedings of the 2nd ACM international symposium on Mobile ad hoc networking \& computing, vol. 1. Long Beach, California, USA: ACM, pp. 95-105 (2001)
22. J Choi, S Bahk, Cell-throughput analysis of the proportional fair scheduler in the single-cell environment. Vehicular Technology, IEEE Transactions on. 56(2), 766-778 (2007)

23. S Ryoo, C Joo, S Bahk, Spectrum allocation with beamforming antenna in het-erogeneous overlaying networks, in Personal Indoor and Mobile Radio Communications (PIMRC), 2010 IEEE 21st International Symposium on, vol. 1. Istanbul, Turkey: IEEE, pp. 1150-1155

doi:10.1186/1687-1499-2012-160

Cite this article as: Ryoo et al:: A decentralized spectrum allocation and partitioning scheme for a two-tier macro-femtocell network with downlink beamforming. EURASIP Journal on Wireless Communications and Networking 2012 2012:160.

\section{Submit your manuscript to a SpringerOpen ${ }^{\circ}$ journal and benefit from:}

- Convenient online submission

- Rigorous peer review

- Immediate publication on acceptance

- Open access: articles freely available online

- High visibility within the field

- Retaining the copyright to your article

Submit your next manuscript at $\gg$ springeropen.com 